\title{
Los caminos actuales de la docencia universitaria
}

\section{Susan Francis Salazar}

Universidad de Costa Rica

-....... onferencia dictada por la Doctora Susan Francis Salazar durante la V Jornada de Innovación Educativa "Docencia, investigación e innovación en la educación superior: una visión integral y prospectiva", realizada en la Universidad Nacional Autónoma de Honduras.

En el tema de la docencia universitaria probablemente uno de los hallazgos más importantes es el hecho de que no es posible pensar en innovación educativa en educación superior sin considerar a los docentes universitarios. Pero además en este marco, quienes apoyamos su desarrollo y actualización docente estamos necesariamente obligados a fundamentarnos en dos grandes pilares: uno es la investigación y otro es la indagación; ambos en el ámbito de la educación universitaria.

En efecto, la universidad no se puede caracterizar de la misma manera que un centro de educación secundaria, por ejemplo. Esto implica que profesores, asesores docentes y gestores académicos requieren trabajar procesos de investigación e indagación en contextos de edu- cación superior en los cuales surjan categorías pedagógicas, curriculares y didácticas que puedan proyectarse de manera pertinente en el ámbito universitario. Este es un esfuerzo sustantivo y fundamental al hablar de innovación educativa en la docencia universitaria.

El recorrido de los actuales caminos de la docencia universitaria conlleva a pensar en las rutas históricas por las que se ha transitado. Por ello, un elemento que me parece importante detallar es que no podemos desligar el ámbito histórico de la docencia universitaria. En la Universidad de Costa Rica (UCR) la docencia universitaria y sus procesos de fundamentación y formación pedagógica tomaron un sitio muy importante desde 1973. E1 estudiantado de aquella época señaló la existencia de importantes limitaciones a la hora de ser formados, porque los profesores no sabían "dar clases", sabían mucho del tema pero no tenían una adecuada metodología docente para trabajar. Entonces la UCR, en el marco de su Tercer Congreso Universitario, crea dos instancias: una que se llama Centro de Evaluación Académica (CEA) ubicada en la Vicerrectoría de Docencia y que se encarga de todos los procesos de diseño curricular y evaluación del desempeño docente y el Centro de Docencia Universitaria, que más tarde se convirtió en el Departamento de Docencia Universitaria (DEDUN), cuya tarea es la formación pedagógica del personal docente de esta universidad.

Para cumplir su tarea, el DEDUN desarrolla cuatro etapas históricas que me permiten entender los caminos de la docencia hacia la innovación. La primera etapa la llamaría: de los métodos docentes, en esta etapa el DEDUN se concentra en la implementación de cursos de formación instrumental, los cuales focalizaban los métodos y técnicas didácticas. No obstante, aunque se presentaron una serie de elementos -algunos propios de la educación superior- también hubo discursos vinculadas con los ámbitos de la educación secundaria que se posicionaron en el aula universitaria con grandes limitaciones.

En la segunda etapa se da una promoción del papel crítico de la docencia, así el DEDUN 
fundamenta su quehacer a partir de la concepción de la docencia desde el punto de vista del compromiso social y el papel que juega el docente $y$ la universidad en la sociedad. Esto generó un importante espacio para la reflexión docente, más allá del tecnicismo, se propuso una docencia crítica que a partir de su conciencia educativa reconoce la acción formativa como una acción transformadora. Sin embargo, lo anterior generó que elementos de carácter didáctico se diluyeran. Finalmente, la tercera etapa, inicia en un proceso de fundamentación pedagógica y diríamos que es el esfuerzo más importante porque es aquí cuando se reconoce la necesidad de hacer investigación para poder aportar y apoyar al profesorado universitario en sus procesos de innovación. En la actualidad el DEDUN fundamenta su acción desde un desarrollo pedagógico que incorpora una serie de menciones investigativas que generan categorías más pertinentes para el ámbito universitario; por ello, apoyan al docente universitario en la comprensión de su papel pedagógico, desde el cual puede tomar decisiones que le permitan desarrollar innovación educativa.

Desde estos procesos investigativos y de indagación compartimos con ustedes los siguientes:

Se puede iniciar cuestionando: ¿Por qué pensar la docencia universitaria como un ámbito investigativo? ¿Quién es el docente universitario? ¿Es posible extrapolar las condiciones de otros niveles educativos al universitario? Pues bien, los procesos de indagación nos muestran que el docente universitario cumple una función sustantiva pues construye la relación de la universidad con la sociedad, las responsabilidades que asumen los académicos son significativas, pues no solo se encargan de la formación profesional, sino también contribuyen con la formación de capacidades de ciudadanía y de desarrollo disciplinar y profesional, en otras palabras los docentes universitarios participan de la posibilidad de que personas que conforman esta sociedad puedan llevarla a un desarrollo humano con más equidad y más posibilidades de justicia social. Es tan fuerte la relación universidad-sociedad que en Centroamérica el 80\% del desarrollo de la investigación es producido por las universidades y son estas quienes tienen la amplia responsabilidad en desarrollar mejores caminos científicos, tecnológicos y sociales.

Asimismo se encuentra que el docente universitario es el encargado de la calidad de los procesos universitarios. En la época actual el docente universitario está más que nunca interpelado porque debe ser una instancia de calidad, por ejemplo casi todas las agencias de acreditación tienen al profesorado como uno de los factores o categoría que hay que analizar y evaluar para saber si realmente la carrera es acreditada o no, si se forma, si sabe, si tiene un buen currículo vitae; el docente universitario es factor claro de calidad, y eso implica que son requeridos, pero al mismo tiempo exigidos a ser mejores todos los días.
¿Cómo llegar a ser mejores todos los días?, ¿Cómo llegar a hacer innovación?, una de las posibilidades es la formación para la docencia en educación superior y una de las nociones más importantes que el DEDUN ha trabajado e investigado es ¿Cómo formar? De nuevo no puede extrapolarse las mismas categorías de formación de docentes de otros niveles al de los docentes universitarios. Si se considera que un docente universitario es una persona que tiene un título universitario, esto significa que ya cuenta con un nivel de formación superior, ya tiene un antecedente de grado profesional. Por lo tanto, ¿Qué características debería tener esa formación? Cualquier esfuerzo para generar procesos de formación docente universitaria requiere ser consistente a la actividad del docente universitario, la única posibilidad para que el docente universitario se acerque a la innovación es partir de la investigación en el contexto universitario. Estos procesos de investigación son los que permiten establecer que para un desarrollo de docencia universitaria de calidad se requiere formación.

Acerca de la palabra formar existe una serie de imprecisiones muy interesantes, el DEDUN tomó como base a Gadamer para aproximarse conceptualmente a este concepto y así precisarlo, así se asume la formación como el proceso de trascendencia de la persona, es decir, cuando se forma se colabora para que las personas superen su estado actual y lleguen a un estado mejor, eso hace que se tenga la responsabi- 
lidad de lograr que esa persona sea mejor. Lo mejor podría generar susceptibilidad, lo mejor lo indica la comunidad académica que representa la universidad, lo mejor es una decisión de proyecto educativo, por tanto es una decisión política.

Básicamente un docente universitario es inicialmente persona y es persona con valores, actitudes y capacidades que le permiten comunicarse con otros. Esta capacidad de interacción personal o la capacidad comunicativa es una característica de un profesorado excelente, además el docente es una persona con capacidad y manejo disciplinar distinto a otros; en otras palabras es capaz de establecer cuáles son los obstáculos epistemológicos que tiene una persona para aprender algo, maneja la estructura de esas teorías, el docente universitario está además preparado para vincularse con un contexto donde relaciona la teoría con la práctica y finalmente tiene la capacidad investigativa y de desarrollo del conocimiento no solo de su disciplina, sino de los ámbitos académicos y profesionales.

La docencia es una actividad educativa pero para desarrollar innovaciones educativas se debe construir un saber pedagógico y formalizar el saber pedagógico que ya se tiene. Por qué hablar de pedagogía en el ámbito universitario. Es un término que en los últimos años se ha clarificado bastante, actualmente se reconoce que su sentido etimológico planteaba un camino hacia la humanización, la palabra pedagogía correspondía a un proyecto helénico sobre cómo hacer que sus ciudadanos fueran más humanos, entonces, en realidad la metáfora de camino a la humanización es lo que explica la idea del camino de la pedagogía. En ese sentido para poder trabajar en innovación educativa era necesario para el DEDUN investigar cómo se construía esa pedagogía en el ámbito universitario.

Las primeras aproximaciones a la dimensión pedagógica el DEDUN las concretó en las acciones de comprensión y gestión curricular, la toma de decisiones pedagógicas y la comprensión del saber en objetos de aprendizaje.

\section{Comprensión}

y gestión curricular

En principio una de las tareas más importantes a nivel de innovación es que el profesorado pueda comprender y gestionar el currículo, ello implica que se identifique ¿Cuál es el objeto de la disciplina o las áreas de acción de la profesión y como se sitúan dentro de un contexto? y ¿Qué implicaciones tienen dentro de ese contexto? Supone, que el docente universitario tenga el conocimiento para hacer el diagnóstico acerca de la formación de las personas, pero además, significa que requiere establecer las principales tareas, funciones y problemas a los que se va a enfrentar el profesional graduado. Por lo tanto, dentro de los procesos de innovación educativa el docente es capaz de establecer las mejores habilidades, competencias y capacidades que se pueden desarrollar en sus cursos, pero también supone que es capaz de darle seguimiento curricular de tal manera que pueda establecer la secuencia de experiencias de aprendizaje que vivirá el estudiantado.

\section{La capacidad de tomar decisiones pedagógicas}

En el quehacer docente muchas de sus decisiones están en vínculo directo con lo pedagógico, como mejorar este proceso de toma de decisiones también es susceptible de ser formado. Para una adecuada toma de decisiones pedagógicas el docente universitario requiere capacidad para analizar

29 2015

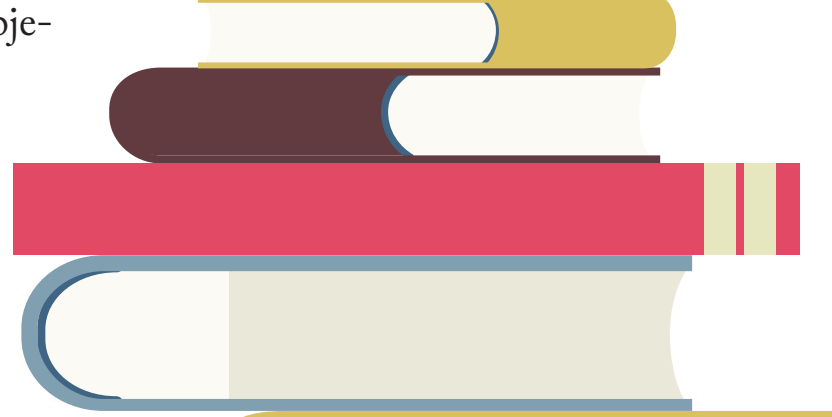


los comportamientos formales que hay que propiciar dentro del estudiantado y la universidad; en particular cómo desarrollar ciertos aprendizajes: Por ejemplo para tomar decisiones pedagógicas un docente requiere preguntarse ¿Cómo se promueve el pensamiento crítico? ¿Cómo se logra que los estudiantes adquieran determinadas habilidades que les permita desarrollar pensamiento crítico? Bueno esto hace necesario, entre otras $\mathrm{co}^{-}$ sas, comprender cómo se desarrolla el pensamiento crítico tomando en cuenta quienes son nuestros estudiantes y su forma de aprender.

Si se toma la referencia de la Organización Mundial de la Salud, OMS, que declara que la finalización de la juventud es

los veinticinco años, la docencia universitaria en su mayoría interactuaría con adolescentes, quienes todavía tienen necesidades de carácter psicológico que nos les permiten llegar a tomar decisiones profesionales, por lo tanto, ¿Cómo se logra que los estudiantes adquieran estos aprendizajes? ¿Cómo aprenden los adolescentes tardíos? ¿Cómo aprenden los adultos jóvenes? También supone preguntarnos qué secuencias, qué experiencias se requieren para aprender, de nuevo: si se requiere el aprendizaje de heurísticas para resolver problemas ¿Cuál es el procedimiento para enseñar la resolución de problemas? ¿Cómo pueden aprender a identificar los niveles algorítmicos, o los niveles de contingencia o bien, los niveles de acción situada, todos, para resolver problemas?

e-xposición
Transformaciones del saber disciplinar/profesional en saberes para ser aprendidos por otros. Como consecuencia de la capacidad de toma de decisiones pedagógicas se reconoce también, que una de las facetas más importantes en el saber pedagógico de los profesores universitarios es la capacidad para transformar los saberes disciplinares o profesionales en saberes que son aptos para ser aprendido por otros. Ese proceso de transformación del saber ha sido estudiado por diferentes programas de investigación en el mundo, uno de los más conocidos es el Programa de Conocimiento Docente, cuyo mayor representante es Lee Shulman quien planteó que todo buen docente cuenta con un conocimiento pedagógico del contenido, algunos exponen esta capacidad como Transposición didáctica concepto desarrollado por los franceses en el esfuerzo de explicar cómo se aprenden las matemáticas.

Las representaciones por tanto surgen de la capacidad de conocimiento que tiene el docente de la disciplina/profesión y de sus articulaciones con los criterios epistemológicos. Las disciplinas (y las profesiones en el entendido de que son ámbitos interdisciplinares) tienen dos dimensiones: la sustantiva y la sintáctica. La primera conjuga todas las teorías y categorías conceptuales que explican su contenido disciplinario y profesional y la dimensión sintáctica es la que incluye los criterios y mecanismos para dar validez a los saberes que surgen, es esta última la que permite construir los criterios pedagógicos para lograr establecer cuáles son las mejores transformaciones, metáforas, analogías, otras para lograr que otras personas aprendan la dimensión sustantiva. Una ilustración de lo anterior lo vemos en una wiki, esta estrategia no funciona igual cuando la aplicamos para aprender algo en Química que en Psicología, son dos cosas completamente distintas aunque la técnica este ahí y sea la misma.

Bajo estas tres características del saber pedagógico en la docencia universitaria es necesario reconocer que la innovación docente tiene implicaciones diversas:

- Primero es necesario una ruptura de ciertas nociones:

- La docencia no es manejar técnicas didácticas, docencia es poder crear escenarios y espacios para que la gente pueda trascender, pueda formarse pueda ponerse en contacto con las dimensiones disciplinares o profesionales.

- La docencia no es conocer todo acerca de un tema, sería necesario complementarlo con un conocimiento pedagógico del contenido que le permita generar las mejores representaciones pedagógicas para aprender.

- La docencia no es transmisión de conocimientos, el conocimiento lo construye el estudiantado en la interacción que se genera en los escenarios creados y promovidos por los estudiantes.

- La docencia no es informar, la información es transmitida por diversos medios: televisión, radio, Internet..., la docencia 
promueve la construcción de conocimientos y para esto es necesario aprender, interactuar con la información, cuestionarla, analizarla, crear nueva información.

- Es urgente variar los roles y las funciones que tradicionalmente se le han consignado a los docentes: esto supone dejar atrás la imagen del docente que informa y se traslada al docente mediador, quien pone en contacto los estudiantes con distintos saberes.

- La innovación es innovación porque se interpreta desde el contexto en el que surge: atiende una necesidad y un problema que surge como resultado de necesidades específicas. La misma innovación podría no ser pertinente en otros contextos.

- El papel del docente como un agente cultural lo involucra como un dinamizador de los procesos de interacción con los distintos discursos y saberes. Cumple su función educativa en un contexto de prácticas y medios, por eso es un mediador entre los saberes socioculturales y los procesos de aprendizaje de estos saberes. $\mathrm{Su}$ desafío mayor es aproximarse a la comunicación pedagógica no sólo entre docente y estudiantes sino también entre los mismos estudiantes y otros agentes culturales.

Desde la Universidad de Costa Rica, el DEDUN consideró que no se buscaba formar pedagogos, pero sí un profesional apto para tomar decisiones pedagógicas. El DEDUN promueve en sus actividades de desarrollo académico que el profesorado sea su propio sujeto de indagación y aquí es donde empezamos a ver que la indagación y la investigación son las fuentes para la innovación. $\mathrm{Si}$ queremos innovar tenemos que convertirnos en sujetos investigadores de nuestras propias prácticas, en las cuales los puntos de partida: son la cotidianidad. Son escenarios reales donde no todo resulta, de ahí que la indagación de las propias experiencias generen $\mathrm{ca}^{-}$ tegorías pedagógicas posibles de compartir y de analizar en el ámbito universitario.

Sobre esta base el DEDUN reconoce que existen lecciones aprendidas en el marco de la formación docente universitaria, entre ellas, las más significativas son la armonización de significados, esto es la necesidad de contar con una plataforma que permita el intercambio y la interacción entre los mismos docentes y los asesores pedagógicos, terminologías educativas para precisar y no generar ambigüedades. Otra lección es la importancia de análisis de los modelos docentes, sobre todo de las características que logran generar aprendizajes en el estudiantado, pero también de aquellos que se convierten en dilemas porque no tienen una sola forma de resolver, o porque sus marcos de referencia y valores las hace complejas. Finalmente se hace fundamental que el profesorado reconozca que existe una consustancialidad de los saberes pedagógicos con los disciplinares/profesionales que explica las maneras en las cuales se desarrollan los actos educativos en sus unidades académicas y en sus tradiciones.
Bajo estas lecciones el DEDUN promueve el concepto de la Docencia Estratégica, esto es la idea de que el docente es capaz de movilizar capacidades y recursos en contextos de manera consciente, siempre indagando sobre su propia práctica y teniendo claridad disciplinar en el manejo de conceptos, identificación de los obstáculos epistemológicos, la proyección en los contextos reales y la identificación de recursos para el aprendizaje (de aquí el valor de las tecnologías de información y comunicación en la actualidad).

Las TIC que es una de las dimensiones que por lo general está asociada con los procesos de innovación porque en ella hay un re-posicionamiento de la relación pedagógica, es decir el estudiante y el docente se convierten en entes activos, la innovación no sucede porque el recurso se implementó, sino por la generación de actividades entre estas dos instancias a fin de potenciar la relación pedagógica.

Con esto, el DEDUN desarrolla su actuar desde procesos de fundamentación pedagógica a partir de investigación y desarrollo de innovaciones como asesores pedagógicos, con ello de ello resultó la definición de un modelo pedagógico y un enfoque formativo desde las bases humanas del desarrollo. No cabe la menor duda de que los aportes neurológicos recientes, aunados a un importante desarrollo de la antropología pedagógica proveen insumos con mayor sentido a la hora de proyectarlos en los espacios humanos, sobre todo los educativos. 
Como consecuencia de lo anterior, los procesos formativos para la docencia universitaria de la UCR, desde el DEDUN, se basan en el proceso humano, comunicativo y el de modelaje donde el profesorado aprende a analizar, a resolver problemas, a criticar a partir del modelo que tienen en frente. Finalmente a las tecnologías de información y comunicación les damos un sitio fundamental ya que es impensable el aprendizaje y los espacios educativos sin tecnología, no obstante, el abordaje es crítico, desde las bases humanas del desarrollo, es posible lograr aprendizajes mediados, pero las tecnologías deben estar asumidas desde un enfoque pedagógico, pues si bien permiten magnificar los resulta- dos de la actividad humana, también pueden magnificar lo tradicional, y eso no es precisamente lo que se busca con su incorporación; reitero en ellas el valor está en que hay un reposicionamiento de la relación pedagógica.

La dinámica del DEDUN ha reconocido que los procesos de innovación docente son un espacio complejo, interdisciplinar que requiere la participación diversa, de ahí que existen focos de atención, temporales, en el entendido que en la emergencia de la construcción del conocimiento las preguntas cambian. En la actualidad reconocemos la importancia de indagar y fundamentarnos desde la relación de cerebro-cultura: el aporte de las ciencias cognitivas y los elementos antropológicos para comprender las construcciones culturales y la inclusión de la diversidad en el ámbito universitario. Asimismo el papel de las tecnologías de la información y comunicación en la redefinición de los espacios del aprendizaje. Por ello, hemos concebido el trabajo en equipo con especialistas de otras áreas y el trabajo en redes: los caminos de la docencia universitaria para lograr la innovación requieren procesos de indagación e investigación dinámicos y la sistematización de experiencias para socializar con otros docentes. 